\title{
Functions Equivalent to Borel Measurable Ones
}

\author{
by
}

\author{
Andrzej KOMISARSKI, Henryk MICHALEWSKI and Paweł MILEWSKI \\ Presented by Czestaw RYLL-NARDZEWSKI
}

Summary. Let $X$ and $Y$ be two Polish spaces. Functions $f, g: X \rightarrow Y$ are called equivalent if there exists a bijection $\varphi$ from $X$ onto itself such that $g \circ \varphi=f$. Using a theorem of J. Saint Raymond we characterize functions equivalent to Borel measurable ones. This characterization answers a question asked by M. Morayne and C. Ryll-Nardzewski

1. Introduction. Let $X$ and $Y$ be two sets. Following Szpilrajn ([12]), we say that functions $f, g: X \rightarrow Y$ are equivalent if there exists a bijection $\varphi$ from $X$ onto itself such that $g \circ \varphi=f$. It is straightforward that $f$ and $g$ are equivalent if and only if $\left|f^{-1}(\{y\})\right|=\left|g^{-1}(\{y\})\right|$ for every $y \in Y$.

Let $X$ be a Polish (i.e. separable, completely metrizable) topological space and let $\mathcal{B} \subset \mathcal{P}(X)$ be the family of Borel subsets of $X$. For a $\sigma$-ideal $\mathcal{I} \subset \mathcal{P}(X)$ we say that a function $g: X \rightarrow Y$ is $\sigma(\mathcal{B}, \mathcal{I})$-measurable if $g^{-1}(U)$ is an element of the smallest $\sigma$-algebra containing $\mathcal{B}$ and $\mathcal{I}$ for any open set $U \subset Y$. In [9] Morayne and Ryll-Nardzewski proved that if a $\sigma$-ideal $\mathcal{I} \subset \mathcal{P}(X)$ contains a set of size continuum and admits a Borel base, which is true in particular for the ideal of meager sets and the ideal of Lebesgue measure null-subsets of the interval $[0,1]$, then a given function $f: X \rightarrow \mathbb{R}$ is equivalent to some $\sigma(\mathcal{B}, \mathcal{I})$-measurable function $g: X \rightarrow \mathbb{R}$ if and only if $\left\{y \in \mathbb{R}: f^{-1}(\{y\}) \neq \emptyset\right\}$ contains a topological copy of the Cantor space or there exists $y \in \mathbb{R}$ such that $\left|f^{-1}(\{y\})\right|=2^{\aleph_{0}}$. They also asked if there is a similar characterization of functions equivalent to Borel measurable ones.

We answer this question using a recent result of J. Saint Raymond ([11]). The result of Morayne and Ryll-Nardzewski was proved by Kysiak for some $\sigma$-ideals not admitting a Borel base, in particular for the ideal of Marczewski null sets and the ideal of completely Ramsey null sets ([7]). Kwiatkowska ([6])

2010 Mathematics Subject Classification: Primary 54H05; Secondary 03E15, 54C10.

Key words and phrases: coanalytic sets, equivalent functions, bimeasurable functions. 
proved that a function $f:[0,1] \rightarrow[0,1]$ is equivalent to a continuous function if and only if it fulfills certain requirements related to the Darboux property alongside with the descriptive set-theoretical requirement that the set $\left\{x \in[0,1]:\left|f^{-1}(x)\right|=2^{\aleph_{0}}\right\}$ is analytic.

2. Notation. All spaces considered are separable and metrizable. The Baire space, $\mathcal{N}$, is defined as the infinite countable product of $\mathbb{N}$, equipped with the Tikhonov topology. Let $X$ be a Polish space. A set $A \subset X$ is analytic if it is a continuous image of $\mathcal{N}$. A set $C \subset X$ is coanalytic if its complement $X \backslash C$ is analytic.

Given a subset $A \subset X \times Y$ we define $A_{x}=\{y \in Y:(x, y) \in A\}$ and $A^{y}=\{x \in X:(x, y) \in A\}$.

Let $\mathbb{N}<\mathbb{N}$ be the set of all finite sequences of the natural numbers. A nonempty subset $T \subset \mathbb{N}^{<\mathbb{N}}$ is a tree if $\forall n \forall k \leq n\left[\left(t_{0}, \ldots, t_{n-1}\right) \in T \Rightarrow\right.$ $\left.\left(t_{0}, \ldots, t_{k-1}\right) \in T\right]$. The space $\operatorname{Tr} \subset 2^{\mathbb{N}<\mathbb{N}}$ is the set of all trees endowed with the topology inherited from $2^{\mathbb{N}<\mathbb{N}}$, it is homeomorphic to the Cantor set. Let $T$ be a tree. We say that $\left(x_{0}, x_{1}, \ldots\right)$ is a branch of $T$ if for every $n \in \mathbb{N}$ we have $\left(x_{0}, x_{1}, \ldots, x_{n}\right) \in T$. Denote by WF $\subset \operatorname{Tr}$ the set of all well-founded trees (i.e. having no branches), and by UB $\subset \operatorname{Tr}$ the set of all trees with exactly one branch.

We will need the following lemma which is a known generalization of the Lusin Unicity Theorem ([1, 18.11]). We include a proof since we have been unable to find a direct reference. Our method of proof is similar to a method used by Z. Koslova in [3].

Lemma 1. For any Borel subset $B \subset X \times Y$ of the product of Polish spaces $X, Y$, the sets $B(n)=\left\{x \in X:\left|B_{x}\right|=n\right\}$ are coanalytic for $n=$ $0,1, \ldots, \aleph_{0}$.

Proof. Being a complement of the projection of $B$ onto $X$, the set $B(0)$ is coanalytic. The Lusin Unicity Theorem guarantees that $B(1)$ is coanalytic. Let $2 \leq n<\aleph_{0}$ and let $U_{1}, U_{2}, \ldots$ be a basis of $Y$. Put $V_{i}=X \times U_{i}$, $i=1,2, \ldots$. Since

$$
\begin{aligned}
x \in B(n) \Leftrightarrow \exists & k_{1}, \ldots, k_{n}\left[\left(\forall i \neq j V_{k_{i}} \cap V_{k_{j}}=\emptyset\right)\right. \\
& \left.\wedge\left(\forall i \leq n\left|\left(B \cap V_{k_{i}}\right)_{x}\right|=1\right) \wedge\left(\left(B \backslash \bigcup_{i=1}^{n} V_{k_{i}}\right)_{x}=\emptyset\right)\right],
\end{aligned}
$$

the set $B(n)$ is coanalytic.

Consider now the case $n=\aleph_{0}$. Let $f: F \rightarrow B$ be a continuous bijection defined on a closed set $F \subset \mathcal{N}$ (cf. [1, 13.7]). Put $G=\{(x, y, z): f(z)=$ $(x, y)\} \subset X \times(Y \times \mathcal{N})$. For a given $x \in X$ the formula $y \mapsto\left(y, f^{-1}(x, y)\right)$ defines a bijection between $B_{x}$ and $G_{x}$. Hence it is sufficient to show that 
$G\left(\aleph_{0}\right)=\left\{x \in X:\left|G_{x}\right|=\aleph_{0}\right\}$ is coanalytic. Note that $G$ is closed and in particular all vertical sections of $G$ are closed. Hence we may assume that $B_{x}$ is closed for every $x \in X$ (we may replace $B$ with $G$ ). The section $B_{x}$ has infinitely many isolated points iff $\forall m \in \mathbb{N} \exists k_{1}, \ldots, k_{m}\left[\left(\forall i \neq j V_{k_{i}} \cap V_{k_{j}}=\emptyset\right) \wedge\left(\forall i=1, \ldots, m\left|\left(B \cap V_{k_{i}}\right)_{x}\right|=1\right)\right]$.

Hence the lemma follows from the observation that

$x \in B\left(\aleph_{0}\right) \Leftrightarrow B_{x}$ is countable and has infinitely many isolated points (the set $\left\{x \in X:\left|B_{x}\right| \leq \aleph_{0}\right\}$ is coanalytic, cf. [1, Theorem 29.19]).

3. Complete pairs of disjoint coanalytic sets. In this section we deal with pairs of disjoint coanalytic sets. For short we call them just pairs. Following Louveau and Saint Raymond (see [8]) we introduce the notion of a reduction of pairs and the notion of a complete pair (called in [8] une pair reductrice pour $\Gamma$ ).

Let $X$ and $Y$ be Polish spaces and let $(A, B)$ and $(C, D)$ be pairs in $X$ and $Y$ respectively. We say that a function $r: X \rightarrow Y$ reduces $(A, B)$ to $(C, D)$ if for any $x \in X$,

$$
x \in A \Leftrightarrow f(x) \in C \text { and } \quad x \in B \Leftrightarrow f(x) \in D .
$$

We say that $r$ is a reduction of $(A, B)$ to $(C, D)$.

A pair $(C, D)$ in a Polish space $Y$ is complete (see [8, a definition before Theorem 9] and Definition 1 in [11]) if for any pair $(A, B)$ in the Baire space there exists a continuous reduction of $(A, B)$ to $(C, D)$. We will need the following result of Saint Raymond:

Theorem 2 ([11, Theorem 23]). The pair (WF, UB) is complete.

Note that since any Polish space $X$ can be embedded into the Baire space by means of a Borel measurable and bijective function, if $(C, D)$ is a complete pair and $(A, B)$ is a pair in $X$, then there exists a Borel measurable function reducing $(A, B)$ to $(C, D)$ (it is the superposition of the Borel measurable embedding and the reduction). Hence we obtain

Corollary 3. Let $A, B$ be coanalytic and disjoint subsets of a Polish space $Y$. Then there exists a Borel function $f: Y \rightarrow \operatorname{Tr}$ such that $x \in A$ if and only if $f(x) \in \mathrm{WF}$ and $x \in B$ if and only if $f(x) \in \mathrm{UB}$.

Using a method of [2] one can prove that if every pair $(A, B)$ in every Polish space can be reduced to $(C, D)$ by a Borel measurable function, then the pair $(C, D)$ is complete.

4. Construction. For a given countable family of disjoint, coanalytic subsets of a Polish space $Y$ indexed by the numbers $\left\{0, \ldots, \aleph_{0}\right\}$, we will construct (Corollary 8) a Borel function on a Polish space $X$ such that the 
coanalytic set with index $n \in\left\{0, \ldots, \aleph_{0}\right\}$ is equal to the set of points with all preimages of cardinality $n$. Furthermore, we will prove that the function may be chosen to be of the first Baire class.

Lemma 4. Let $Y$ be a Polish space and let $B(0), B(1), \ldots, B\left(\aleph_{0}\right)$ be subsets of $Y$. The following conditions are equivalent:

(i) For every uncountable Polish space $X$ there exists a Borel measurable function $f: X \rightarrow Y$ such that $B(n)=\left\{y \in Y:\left|f^{-1}(y)\right|=n\right\}$ for every $n \in\left\{0,1, \ldots, \aleph_{0}\right\}$.

(ii) For every uncountable Polish space $X$ there exists an uncountable Borel set $B \subset X \times Y$ such that $B(n)=\left\{y \in Y:\left|B^{y}\right|=n\right\}$ for every $n \in\left\{0,1, \ldots, \aleph_{0}\right\}$.

(iii) There exists an uncountable closed set $F \subset \mathcal{N} \times Y$ such that $B(n)=$ $\left\{y \in Y:\left|F^{y}\right|=n\right\}$ for every $n \in\left\{0,1, \ldots, \aleph_{0}\right\}$.

(iv) There is a Polish space $X$ and an uncountable Borel set $B \subset X \times Y$ such that $B(n)=\left\{y \in Y:\left|B^{y}\right|=n\right\}$ for every $n \in\left\{0,1, \ldots, \aleph_{0}\right\}$.

Proof. Since the graph of a Borel measurable function $f: X \rightarrow Y$ is a Borel subset of $X \times Y$, obviously (i) implies (ii). Now, let $X$ be an uncountable Polish space and let $B \subset X \times Y$ be an uncountable Borel set. To show that (ii) implies (iii), fix a closed subset $A$ of $\mathcal{N}$ and a continuous bijection $g: A \rightarrow B$ (cf. [1, 13.7]) and define $F=\{(z, y) \in \mathcal{N} \times Y: z \in A$, $\left.y=\pi_{Y} \circ g(z)\right\}$, where $\pi_{Y}: X \times Y \rightarrow Y$ is the projection. Then $F$ satisfies (iii), because for a given $y \in Y$ the formula $x \mapsto g^{-1}(x, y)$ defines a bijection between $B^{y}$ and $F^{y}$. Clearly (iii) implies (iv). Finally, to prove (iv) $\Rightarrow$ (i) assume that $X$ and $B \subset X \times Y$ satisfy (iv) and $X^{\prime}$ is an arbitrary uncountable Polish space. Fix a Borel isomorphism $g: X^{\prime} \rightarrow B$ (any two uncountable Borel subsets of Polish spaces are Borel isomorphic, cf. [1, 15.6]). Now we can define $f=\pi_{Y} \circ g$. The proof is complete.

Theorem 5. Let $B(0), B(1), \ldots, B\left(\aleph_{0}\right)$ be subsets of a Polish space $Y$. If $B(0), B(1), \ldots, B\left(\aleph_{0}\right)$ are coanalytic, pairwise disjoint and $\bigcup_{n=0, \ldots, \aleph_{0}} B(n)$ $\neq Y$ or $Y \backslash B(0)$ is uncountable then $B(0), B(1), \ldots, B\left(\aleph_{0}\right)$ satisfy conditions (i)-(iv) of Lemma 4 .

If $B(0), B(1), \ldots, B\left(\aleph_{0}\right) \subset Y$ satisfy one of the conditions of Lemma 4 then they are coanalytic, pairwise disjoint and $Y \backslash B(0)$ is uncountable or $\bigcup_{n=0, \ldots, \aleph_{0}} B(n) \neq Y$.

Proof. Let $B(0), B(1), \ldots, B\left(\aleph_{0}\right)$ be pairwise disjoint coanalytic subsets of a Polish space $Y$. We will prove that condition (iv) of Lemma 4 is fulfilled. To this end we define $B\left(2^{\aleph_{0}}\right)=Y \backslash \bigcup_{n=0, \ldots, \aleph_{0}} B(n)$. The set $B\left(2^{\aleph_{0}}\right)$ is analytic and if it is nonempty, then there exists a continuous surjection $s: \mathcal{N} \rightarrow B\left(2^{\aleph_{0}}\right)$. 
Claim 6. If $B(0)$ and $B(1)$ are two disjoint coanalytic subsets of a Polish space $Y$ then there exists a Borel set $B \subset \mathcal{N} \times Y$ such that $B(0) \subset$ $\left\{y \in Y: B^{y}=\emptyset\right\}, B(1) \subset\left\{y \in Y: B^{y}\right.$ contains exactly one element $\}$.

Proof of the Claim. Due to Corollary 3 there exists a Borel map $r: Y \rightarrow$ Tr reducing the pair $(B(0), B(1))$ to $(\mathrm{WF}, \mathrm{UB})$. Put $B=$ $\{(x, y): x$ is an infinite branch of $r(y)\}$.

By the Claim there exist Borel sets $B_{n} \subset \mathcal{N} \times Y$ such that $\bigcup_{i \leq n} B(i) \subset$ $\left\{y \in Y:\left(B_{n}\right)^{y}=\emptyset\right\}$ and $\bigcup_{n<i \leq \aleph_{0}} B(i) \subset\left\{y \in Y:\left(B_{n}\right)^{y}\right.$ contains exactly one element $\}, n=0,1,2, \ldots$.

If $B\left(2^{\aleph_{0}}\right) \neq \emptyset$ then we define $X=(\mathcal{N} \times \mathcal{N}) \oplus(\mathbb{N} \times \mathcal{N})$ and $B=\{(x, z, y)$ : $s(x)=y\} \oplus \bigcup_{n \in \mathbb{N}}\{n\} \times B_{n}$. If $B\left(2^{\aleph_{0}}\right)=\emptyset$ then we define $X=\mathbb{N} \times \mathcal{N}$ and $B=\bigcup_{n \in \mathbb{N}}\{n\} \times B_{n}$. The set $B$ is uncountable and has all the required properties.

Now, let $Y$ and $B(0), B(1), \ldots, B\left(\aleph_{0}\right) \subset Y$ satisfy condition (iv) of Lemma 4 for a Polish space $X$ and $B \subset X \times Y$. The sets $B(0), B(1), \ldots, B\left(\aleph_{0}\right)$ are pairwise disjoint and by Lemma 1 they are coanalytic. Since $B$ is uncountable, $Y \backslash B(0)$ is uncountable or $\bigcup_{n=0, \ldots, \aleph_{0}} B(n) \neq Y$.

In the next corollary we will need the following

Proposition 7. Let $f: X \rightarrow Y$ be a Borel function between Polish spaces $X$ and $Y$. Then there exist maps of the first Baire class $g: X \rightarrow Y$ and $h: X \rightarrow X$ such that $g=f \circ h$.

Proof. Let $\phi$ be a bijection of the first Baire class between $X$ and the graph of $f$ (see [5. Corollaire, p. 212]). Put $h=\pi_{X} \circ \phi, g=\pi_{Y} \circ \phi$, where $\pi_{X}$ and $\pi_{Y}$ are the projections from $X \times Y$ onto $X$ and $Y$, respectively.

Corollary 8. Let $X$ and $Y$ be Polish spaces. For a function $f: X \rightarrow Y$ the following conditions are equivalent

(i) $f$ is equivalent to a function of the first Baire class,

(ii) $f$ is equivalent to a Borel measurable map,

(iii) $\left|f^{-1}(y)\right| \in\left\{0,1, \ldots, \aleph_{0}, 2^{\aleph_{0}}\right\}$ for every $y \in Y$ and $\left\{y \in Y:\left|f^{-1}(y)\right|\right.$ $=n\}$ is coanalytic for $n=0, \ldots, \aleph_{0}$.

REMARK. Equivalence of (i) and (ii) was proved by Szpilrajn (12, Section 3.1]).

Proof. The equivalence of (ii) and (iii) follows immediately from Theorem 5 and Lemma 4. The implication (i) $\Rightarrow$ (ii) is obvious and (ii) $\Rightarrow$ (i) follows from Proposition 7 .

Note that Corollary 8 gives a full answer to the Morayne and RyllNardzewski's question. 
5. Functions equivalent to bimeasurable or continuous ones. Let $X, Y$ be Polish spaces. Following [10] we will call a function $f: X \rightarrow Y$ bimeasurable if it is Borel and $f[B]$ is Borel for every $B \in \mathcal{B}(X)$. In the previous section, for a given function $f: X \rightarrow Y$ we described conditions which guarantee that $f$ is equivalent to a Borel measurable function. We may consider a more general problem of characterizing those functions which are equivalent to a function from a given class. In this section we consider the problem for two classes of functions:

- Theorem 10 indicates conditions which ensure that $f: X \rightarrow Y$ is equivalent to a bimeasurable function; in Proposition 11 we consider the notion of Borel equivalence of functions and prove that it is connected with the notion of bimeasurability.

- In Theorem 13 we assume that $X=\mathcal{N}$ and formulate necessary and sufficient conditions for $f$ to be equivalent to a continuous function.

First, we recall

Theorem 9 (Purves [10]). A Borel function $f: X \rightarrow Y$ is bimeasurable if and only if for all but countably many $y \in Y$ the fiber $f^{-1}(y)$ is countable.

TheOREM 10. A function $f: X \rightarrow Y$ is equivalent to a bimeasurable one if and only if for all but countably many $y \in Y$ the fiber $f^{-1}(y)$ is countable, the cardinalities of all fibers of $f$ belong to the set $\left\{0,1, \ldots, \aleph_{0}, 2^{\aleph_{0}}\right\}$ and the sets $\left\{y \in Y:\left|f^{-1}(y)\right|=n\right\}$ are Borel for $n=0, \ldots, \aleph_{0}$.

Proof. Necessity follows directly from Theorem 9 and the fact that every uncountable Borel set has cardinality $2^{\aleph_{0}}$. The above condition is also sufficient. By Corollary 8 the function $f$ is equivalent to some Borel measurable function $g$. By Theorem 9 the function $g$ is bimeasurable.

We say that $f, g: X \rightarrow Y$ are Borel equivalent if there is a Borel automorphism $h: X \rightarrow X$ such that $f=g \circ h$. Directly from the definition, for every pair of Borel equivalent functions, if one of them is Borel, then so is the other. The converse is in general false - Proposition 11 gives a wide class of examples of pairs of Borel functions which are equivalent, but not Borel equivalent.

For fixed spaces $Y, X$ and a subset $F \subset Y \times X$, we call a Borel function $u: \pi_{Y}[F] \rightarrow X$ a Borel uniformization of $F$ if $(y, u(y)) \in F$ for every $y \in \pi_{Y}[F]$.

Observation. (a) If $f, g: X \rightarrow Y$ are Borel equivalent, $Y_{0} \subset Y$ and the set $F=\left\{(y, x) \in Y_{0} \times X: f(x)=y\right\}$ has a Borel uniformization, then $G=\left\{(y, x) \in Y_{0} \times X: g(x)=y\right\}$ has one as well (take the superposition of any uniformization of $F$ and an equivalence of $f$ and $g$ ). 
(b) For every uncountable Borel set $B$ :

- There exists a Borel function $\psi: B \rightarrow \mathcal{N}$ with all fibers uncountable such that $\{(y, x) \in \mathcal{N} \times B: \psi(x)=y\}$ has a Borel uniformization $u$. Indeed, if $f: B \rightarrow \mathcal{N} \times \mathcal{N}$ is a Borel isomorphism and $s: \mathcal{N} \rightarrow \mathcal{N} \times \mathcal{N}$ is given by $s(x)=\left(x, y_{0}\right)$ for some $y_{0} \in \mathcal{N}$, then we define

$$
\psi=\pi_{1} \circ f, \quad u=f^{-1} \circ s,
$$

where $\pi_{i}: \mathcal{N} \times \mathcal{N} \rightarrow \mathcal{N}$ is the projection on the $i$ th coordinate $(i=1,2)$.

- There exists a Borel function $\phi: B \rightarrow \mathcal{N}$ with all fibers uncountable such that $\{(y, x) \in \mathcal{N} \times B: \phi(x)=y\}$ does not have a Borel uniformization. Indeed, let $A \subset \mathcal{N} \times \mathcal{N}$ be a Borel set without a Borel uniformization and with all vertical sections uncountable (cf. [1, Exercise 18.17, one solution on p. 360 and another one on p. 365 in notes to 35.1]) and let $f: B \rightarrow A$ be a Borel isomorphism. We define

$$
\phi=\pi_{1} \circ f .
$$

Then for every Borel uniformization $u: \mathcal{N} \rightarrow B$ of the set $\{(y, x) \in$ $\mathcal{N} \times B: \phi(x)=y\}$, the superposition $\pi_{2} \circ f \circ u: \mathcal{N} \rightarrow \mathcal{N}$ would be a Borel uniformization of $A$, a contradiction.

Proposition 11. Let $f: X \rightarrow Y$ be a Borel function. Then the following conditions are equivalent:

(i) every Borel map from $X$ to $Y$ equivalent to $f$ is also Borel equivalent to $f$,

(ii) $f$ is bimeasurable.

Proof. (ii) $\Rightarrow$ (i). Let $g: X \rightarrow Y$ be a Borel map equivalent to $f$ and let $F, G$ be the graphs of $f, g$, respectively. Then the sets

$$
F(n)=\left\{y:\left|f^{-1}(y)\right|=n\right\}, \quad G(n)=\left\{y:\left|g^{-1}(y)\right|=n\right\}
$$

are Borel, $F(n)=G(n)\left(n=1,2, \ldots, \aleph_{0}, 2^{\aleph_{0}}\right)$ and $F\left(2^{\aleph_{0}}\right)=G\left(2^{\aleph_{0}}\right)=\left\{y_{j}\right.$ : $j \in J\}$ for a countable set $J$.

Let $n \leq \aleph_{0}$. For every $y \in F(n)$ the horizontal section $F^{y}$ is of cardinality $n$. By the Lusin-Novikov Theorem $([1,18.10])$ there are Borel functions $f_{n}^{i}: F(n) \rightarrow X, 1 \leq i \leq n$, such that $\bigcup_{i=1}^{n} \operatorname{Graph}\left(f_{n}^{i}\right)=F \cap(X \times F(n))$. Maps $g_{n}^{i}$ are defined in the same way. Let $A_{n}^{i}=f_{n}^{i}[F(n)], B_{n}^{i}=g_{n}^{i}[G(n)]$, $1 \leq n \leq \aleph_{0}, 1 \leq i \leq n$, and let $A_{j}=f^{-1}\left(y_{j}\right), B_{j}=g^{-1}\left(y_{j}\right), j \in J$. Now, let $h_{n}^{i}: A_{n}^{i} \rightarrow B_{n}^{i}, h_{j}: A_{j} \rightarrow B_{j}$ be Borel isomorphisms. Finally, we define the 
required Borel automorphism $h: X \rightarrow X$ to be $h_{n}^{i}$ on $A_{n}^{i}, 1 \leq i \leq n \leq \aleph_{0}$, and $h_{j}$ on $A_{j}, j \in J$.

(i) $\Rightarrow$ (ii). Assume, aiming for a contradiction, that $f$ is not bimeasurable. By Theorem 9 the set $\left\{y \in Y:\left|f^{-1}(y)\right|=2^{\aleph_{0}}\right\}$ is uncountable. Since it is analytic (cf. [1, Theorem 29.19]), it contains a copy $N$ of the Baire space. Let $B=f^{-1}[N]$. If the set $F=\{(y, x) \in N \times B: f(x)=y\}$ does not have a Borel uniformization, then we define $g: X \rightarrow Y$ to be $\psi$ on $B$ and $f$ on $X \backslash B$. If the set $F$ has a Borel uniformization, then we define $g$ to be $\phi$ on $B$ and $f$ on $X \backslash B(\phi, \psi$ are defined in Observation (b) preceding the proposition).

The fibers of $f$ and $g$ have the same cardinality, hence the functions are equivalent. However, according to Observation (a) applied to the set $N \subset Y$, they are not Borel equivalent.

Let $X, Y$ be Polish spaces and $f: X \rightarrow Y$ be a Borel function that is not bimeasurable. The construction in the proof of the implication (i) $\Rightarrow$ (ii), after a minor modification, gives a family of $2^{\aleph_{0}}$ Borel functions which are pairwise Borel inequivalent, but equivalent to $f$.

The rest of the section is devoted to the characterization of the functions which are equivalent to a continuous function from $\mathcal{N}$ to a Polish space $Y$. We call a nonempty subset $B$ of a Polish space $X$ locally uncountable if every nonempty relatively open subset of $B$ is uncountable. We will need the following standard lemma which is a variation of [1, Exercise 7.15$]$ :

Lemma 12. If $B \subset X$ is a locally uncountable Borel set, then there exists a continuous bijection $\psi: \mathcal{N} \rightarrow B$.

Remark. (a) Let $B$ be a Borel and locally uncountable set. We fix a continuous bijection $\psi: \mathcal{N}^{2} \rightarrow B$ given by Lemma $12\left(\mathcal{N}^{2}\right.$ and $\mathcal{N}$ are homeomorphic). Let $D_{0}, D_{1}, \ldots$ be Borel, pairwise disjoint, dense subsets of $\mathcal{N}$ such that $\mathcal{N}=\bigcup_{i=0}^{\infty} D_{i}$. Then the sets $B_{i}=\psi\left[D_{i} \times \mathcal{N}\right], i=0,1, \ldots$, are Borel, pairwise disjoint, locally uncountable and dense in $B$.

(b) Let $A$ be a locally uncountable set. For a given set $B$, if $A \subset B \subset \bar{A}$, then $B$ is also locally uncountable.

TheOREM 13. Let $f: \mathcal{N} \rightarrow Y$ be a Borel function. Then the following conditions are equivalent:

(i) there is a continuous $g: \mathcal{N} \rightarrow Y$ equivalent to $f$,

(ii) there is a continuous $g: \mathcal{N} \rightarrow Y$ Borel equivalent to $f$,

(iii) for every open set $U$ in $Y$, the preimage $f^{-1}[U]$ is empty or uncountable. 
Proof. The implications (i) $\Rightarrow$ (iii) and (ii) $\Rightarrow$ (i) are easy, so we give a proof of (iii) $\Rightarrow$ (ii) only. Let $K$ be a compactification of $\mathcal{N}$ and let $G=$ $\{(x, f(x)): x \in \mathcal{N}\} \subset K \times Y$ be the graph of $f$. Let $A$ be the set of points of $G$ with a countable neighborhood in $G$. Then both $A$ and $Z=$ $\pi_{Y}[A] \backslash\left\{y \in Y:\left|f^{-1}(y)\right|=2^{\aleph_{0}}\right\}$ are countable. Let $B=G \backslash\left(A \cup \pi_{Y}^{-1}[Z]\right)$. Note that $\left|B^{y}\right|=\left|G^{y}\right|=\left|f^{-1}(y)\right|$ for $y \in Y \backslash Z$ and $\left|B^{y}\right|=0$ for $y \in Z$. According to (iii) and because of compactness of $K$, for every $Z$ there is a point $k_{y} \in K$ such that $p_{y}=\left(k_{y}, y\right) \in \bar{B}^{K \times Y} \subset K \times Y$.

The set $B$ is locally uncountable and Borel, hence Remark (a) guarantees that there exist Borel subsets $B_{0}, B_{1}, \ldots$ of $B$ which are pairwise disjoint, locally uncountable, dense in $B$ and such that $B=\bigcup_{n \in \mathbb{N}} B_{n}$.

Since the sets $C_{i}=\left\{p_{y}: i<\left|f^{-1}(y)\right|, y \in Z\right\}$ are countable, the sets $\widetilde{B}_{i}=\left(B_{i} \cup C_{i}\right) \times\{i\}$ are Borel in $K \times Y \times \mathbb{N}$. Moreover, by Remark (b), they are locally uncountable. Let $\psi_{i}: \mathcal{N} \rightarrow \widetilde{B}_{i}$ be continuous bijections given by Lemma 12 Let $\widetilde{B}=\bigcup_{i \in \mathbb{N}} \widetilde{B}_{i}$ and let $\psi: \mathcal{N} \times \mathbb{N} \rightarrow \widetilde{B}$ be defined by $\psi(x, i)=\psi_{i}(x), i \in \mathbb{N}$. For every $y \in Z$ take a bijection $\phi_{y}$ : $\left\{p_{y}\right\} \times\left\{i: i<\left|f^{-1}(y)\right|\right\} \rightarrow G \cap \pi_{Y}^{-1}(y)$. Then $\phi: \widetilde{B} \rightarrow G$ defined to be $\pi_{K \times Y}$ on $\pi_{K \times Y}^{-1}[B]$ and $\phi_{y}$ on the domain of $\phi_{y}$ is a Borel isomorphism. Fix a homeomorphism $k: \mathcal{N} \rightarrow \mathcal{N} \times \mathcal{N}$ and let $\tilde{f}: \mathcal{N} \rightarrow G$ be defined by $\widetilde{f}(x)=(x, f(x))$. Now it is sufficient to define $g: \mathcal{N} \rightarrow Y$ and $h: \mathcal{N} \rightarrow \mathcal{N}$ by

$$
g=\pi_{Y} \circ \phi \circ \phi \circ k, \quad h=k^{-1} \circ \psi^{-1} \circ \phi^{-1} \circ \widetilde{f} .
$$

\section{References}

[1] A. S. Kechris, Classical Descriptive Set Theory, Springer, New York, 1995.

[2] -, On the concept of $\Pi_{1}^{1}$-completeness, Proc. Amer. Math. Soc. 125 (1997), 18111814 .

[3] Z. Koslova, Sur les ensembles plans analytiques ou mesurables B, Bull. Acad. Sci. URSS Sér. Math. 4 (1940), 479-500.

[4] K. Kuratowski, Topologie, Vol. I, Państwowe Wydawnictwo Naukowe, Warszawa, 1966.

[5] -, Sur une généralisation de la notion d'homéomorphie, Fund. Math. 22 (1934), 206-220.

[6] A. Kwiatkowska, Continuous functions taking every value a given number of times, Acta Math. Hungar. 121 (2008), 229-242.

[7] M. Kysiak, Some remarks on indicatrices of measurable functions, Bull. Polish Acad. Sci. 53 (2005), 281-284.

[8] A. Louveau et J. Saint Raymond, Les propriétés de réduction et de norme pour les classes de Boréliens, Fund. Math. 131 (1988), 223-243.

[9] M. Morayne and C. Ryll-Nardzewski Functions equivalent to Lebesgue measurable ones, Bull. Polish Acad. Sci. Math. 47 (1999), 263-265.

[10] R. Purves, On bimeasurable functions, Fund. Math. 58 (1966) 149-157.

[11] J. Saint Raymond, Complete pairs of coanalytic sets, ibid. 194 (2007), 267-281. 
[12] E. Szpilrajn, Sur l'équivalence des suites d'ensembles et l'équivalence des fonctions, ibid. 26 (1936), 302-326.

Andrzej Komisarski

Institute of Mathematics

University of Łódź

Banacha 22

90-238 Łódź, Poland

E-mail: andkom@math.uni.lodz.pl
Henryk Michalewski, Paweł Milewski Institute of Mathematics University of Warsaw

Banacha 2 02-097 Warszawa, Poland E-mail: H.Michalewski@mimuw.edu.pl pamil@mimuw.edu.pl 